\title{
ON THE TOPOLOGICAL CENTRE PROBLEM FOR WEIGHTED CONVOLUTION ALGEBRAS AND SEMIGROUP COMPACTIFICATIONS
}

\author{
MATTHIAS NEUFANG
}

(Communicated by N. Tomczak-Jaegermann)

\begin{abstract}
Let $\mathcal{G}$ be a locally compact, non-compact group (we make the noncompactness assumption, for the most part, simply to avoid trivialities). We show that under a very mild assumption on the weight function $w$, the weighted group algebra $L_{1}(\mathcal{G}, w)$ is strongly Arens irregular in the sense of Dales and Lau; i.e., both topological centres of $L_{1}(\mathcal{G}, w)^{* *}$ equal $L_{1}(\mathcal{G}, w)$. Also, we show that the topological centre of the algebra $\operatorname{LUC}\left(\mathcal{G}, w^{-1}\right)^{*}$ equals the weighted measure algebra $\mathrm{M}(\mathcal{G}, w)$. Moreover, still in the same situation, we prove that every linear (left) $L_{\infty}\left(\mathcal{G}, w^{-1}\right)^{*}$-module map on $L_{\infty}\left(\mathcal{G}, w^{-1}\right)$ is automatically bounded, and even $w^{*}-w^{*}$-continuous, hence given by convolution with an element in $\mathrm{M}(\mathcal{G}, w)$. To this end, we derive a general factorization theorem for bounded families in the $L_{\infty}\left(\mathcal{G}, w^{-1}\right)^{*}$-module $L_{\infty}\left(\mathcal{G}, w^{-1}\right)$. Finally, using this result in the case where $w \equiv 1$, we give a short proof of a theorem due to Protasov and Pym, stating that the topological centre of the semigroup $\mathcal{G}^{\text {LUC }} \backslash \mathcal{G}$ is empty, where $\mathcal{G}^{\text {LUC }}$ denotes the LUC-compactification of $\mathcal{G}$. This sharpens an earlier result by Lau and Pym; moreover, our method of proof gives a partial answer to a problem raised by Lau and Pym in 1995.
\end{abstract}

\section{INTRODUCTION}

Let $\mathcal{G}$ be a locally compact group, and let $w: \mathcal{G} \rightarrow(0, \infty)$ be a weight function, i.e., a positive, continuous function on $\mathcal{G}$ such that $w(s t) \leq w(s) w(t)$ for all $s, t \in \mathcal{G}$; for convenience we shall assume that $w(e)=1$, where $e$ is the neutral element of $\mathcal{G}$. We will consider the following spaces, normed in such a way that multiplication resp. division by the weight becomes an isometry between the unweighted and the

Received by the editors June 26, 2006, and, in revised form, August 31, 2006.

2000 Mathematics Subject Classification. Primary 22D15, 43A10, 43A20, 43A22, 46H40, $54 \mathrm{D} 35$.

Key words and phrases. Locally compact group, weighted group algebra, left uniformly continuous function, Arens product, topological centre, semigroup compactification.

The present work was partly supported by NSERC. This support is gratefully acknowledged. 
corresponding weighted space (whose norm we will denote by $\|\cdot\|_{w}$ ):

$$
\begin{aligned}
L_{1}(\mathcal{G}, w) & =\left\{f \mid w f \in L_{1}(\mathcal{G})\right\}, \\
L_{\infty}\left(\mathcal{G}, w^{-1}\right) & =\left\{f \mid w^{-1} f \in L_{\infty}(\mathcal{G})\right\}, \\
\operatorname{LUC}\left(\mathcal{G}, w^{-1}\right) & =\left\{f \mid w^{-1} f \in \operatorname{LUC}(\mathcal{G})\right\}, \\
\mathrm{C}_{0}\left(\mathcal{G}, w^{-1}\right) & =\left\{f \mid w^{-1} f \in \mathrm{C}_{0}(\mathcal{G})\right\}, \\
\mathrm{M}(\mathcal{G}, w) & =\{\mu \mid w \mu \in \mathrm{M}(\mathcal{G})\} .
\end{aligned}
$$

Here, $\operatorname{LUC}(\mathcal{G})$ is the space of all bounded left uniformly continuous functions $f$ : $\mathcal{G} \rightarrow \mathbb{C}$; i.e., the map $\mathcal{G} \ni x \mapsto l_{x} f \in\left(L_{\infty}(\mathcal{G}),\|\cdot\|_{\infty}\right)$ is continuous, where $\left(l_{x} f\right)(y)=$ $f(x y)(x, y \in \mathcal{G})$. Also, $\mathrm{C}_{0}(\mathcal{G})$ stands for the space of continuous functions on $\mathcal{G}$ that vanish at infinity, and (its dual) $\mathrm{M}(\mathcal{G})$ is the space of all complex Radon measures on $\mathcal{G}$.

We have $L_{\infty}\left(\mathcal{G}, w^{-1}\right)=L_{1}(\mathcal{G}, w)^{*}$ and $\mathrm{M}(\mathcal{G}, w)=\mathrm{C}_{0}\left(\mathcal{G}, w^{-1}\right)^{*}$. For every $y \in \mathcal{G}$, we define $\widetilde{\delta}_{y}:=w(y)^{-1} \delta_{y}$, which is an element of norm one in $\operatorname{M}(\mathcal{G}, w)$.

Before stating our main results, which are concerned with biduals of weighted convolution algebras and topological centres, we shall provide the necessary background on Arens products.

The first (= left) and second (= right) Arens multiplications arising from the convolution product, denoted by "*", in $L_{1}(\mathcal{G}, w)$ are each constructed in three steps via various module actions. For $m, n \in L_{1}(\mathcal{G}, w)^{* *}, h \in L_{1}(\mathcal{G}, w)^{*}$ and $f, g \in$ $L_{1}(\mathcal{G}, w)$ one defines:

$$
\begin{array}{rll}
\langle h \odot f, g\rangle & :=\langle h, f * g\rangle, \\
\langle n \odot h, f\rangle & :=\langle n, h \odot f\rangle, \\
\langle m \odot n, h\rangle & :=\quad\langle m, n \odot h\rangle,
\end{array}
$$

and, similarly,

$$
\begin{aligned}
\langle f . h, g\rangle & :=\langle h, g * f\rangle, \\
\langle h . m, f\rangle & :=\langle m, f . h\rangle, \\
\langle m . n, h\rangle & :=\langle n, h . m\rangle .
\end{aligned}
$$

Then $m \odot n$ and $m . n$ are called the first, resp., second Arens product of $m$ and $n$. A fairly comprehensive exposition of the basic theory of Arens products is given in [16, Section 1.4].

As for the topological centres corresponding to the two Arens products, an excellent source is [11]. We shall only need their definitions, which we recall here:

$$
\begin{aligned}
\mathfrak{Z}_{t}^{(\ell)}\left(L_{1}(\mathcal{G}, w)^{* *}\right):=\left\{m \in L_{1}(\mathcal{G}, w)^{* *} \mid n \mapsto m \odot n \text { is } w^{*}-w^{*}\right. \text {-continuous } \\
\text { on } \left.L_{1}(\mathcal{G}, w)^{* *}\right\}
\end{aligned}
$$

and, similarly,

$$
\begin{aligned}
\mathfrak{Z}_{t}^{(r)}\left(L_{1}(\mathcal{G}, w)^{* *}\right):=\left\{m \in L_{1}(\mathcal{G}, w)^{* *} \mid n \mapsto n . m \text { is } w^{*}-w^{*}\right. \text {-continuous } \\
\left.\quad \text { on } L_{1}(\mathcal{G}, w)^{* *}\right\} .
\end{aligned}
$$

In the following, unless indicated otherwise, we shall always regard $L_{1}(\mathcal{G}, w)^{* *}$ as endowed with the first Arens multiplication, and hence focus on the first topological centre (since in our situation the second Arens product behaves in a perfectly analogous way). 
We will use the fact that, with the natural module operation stemming from the construction of the first Arens product on $L_{1}(\mathcal{G}, w)^{* *}$, the equality $L_{\infty}\left(\mathcal{G}, w^{-1}\right) \odot$ $L_{1}(\mathcal{G}, w)=\operatorname{LUC}\left(\mathcal{G}, w^{-1}\right)$ holds (see the first assertion of Prop. 1.3 in [5]). Hence, a natural module operation of $\operatorname{LUC}\left(\mathcal{G}, w^{-1}\right)^{*}$ on $L_{\infty}\left(\mathcal{G}, w^{-1}\right)$ is given by

$$
\langle m \diamond h, g\rangle=\langle m, h \odot g\rangle \text {, }
$$

where $m \in \operatorname{LUC}\left(\mathcal{G}, w^{-1}\right)^{*}, h \in L_{\infty}\left(\mathcal{G}, w^{-1}\right), g \in L_{1}(\mathcal{G}, w)$. Note that we clearly have $m \diamond h=m^{\sharp} \odot h$, where $m^{\sharp}$ is an arbitrary Hahn-Banach extension of $m$ to $L_{\infty}\left(\mathcal{G}, w^{-1}\right)^{*}$.

The above module action of $\operatorname{LUC}\left(\mathcal{G}, w^{-1}\right)^{*}$ on $L_{\infty}\left(\mathcal{G}, w^{-1}\right)$ induces an action of $\operatorname{LUC}\left(\mathcal{G}, w^{-1}\right)^{*}$ on $\operatorname{LUC}\left(\mathcal{G}, w^{-1}\right)$ (denoted by the same symbol), which in turn gives rise to a Banach algebra product in $\operatorname{LUC}\left(\mathcal{G}, w^{-1}\right)^{*}:$ for $m, n \in \operatorname{LUC}\left(\mathcal{G}, w^{-1}\right)^{*}$ and $f \in \operatorname{LUC}\left(\mathcal{G}, w^{-1}\right)$ one sets

$$
\langle m n, f\rangle:=\langle m, n \diamond f\rangle .
$$

In perfect analogy to the first topological centre $\mathfrak{Z}_{t}^{(\ell)}\left(L_{1}(\mathcal{G}, w)^{* *}\right)$ of $L_{1}(\mathcal{G}, w)^{* *}$ one defines the topological centre of the algebra $\operatorname{LUC}\left(\mathcal{G}, w^{-1}\right)^{*}$ to be

$$
\begin{aligned}
\mathfrak{Z}_{t}\left(\operatorname{LUC}\left(\mathcal{G}, w^{-1}\right)^{*}\right):=\left\{m \in \operatorname{LUC}\left(\mathcal{G}, w^{-1}\right)^{*} \mid\right. \\
\left.n \mapsto m n \text { is } w^{*}-w^{*} \text {-continuous on } \operatorname{LUC}\left(\mathcal{G}, w^{-1}\right)^{*}\right\} .
\end{aligned}
$$

In the sequel, we shall denote by $\kappa(\mathcal{G})$ the compact covering number of the group $\mathcal{G}$, i.e., the least cardinality of a compact covering of $\mathcal{G}$. For the sake of brevity, we further introduce the following terminology (cf. also [1]).

Definition 1.1. (i) A subset $S$ of $\mathcal{G}$ will be called dispersed if $S$ is not contained in any union of a family of compact subsets of $\mathcal{G}$, the family having cardinality strictly less than $\kappa(\mathcal{G})$.

(ii) For a subset $S \subseteq \mathcal{G}$, we say that the weight $w$ is diagonally bounded on $S$ if we have:

$$
\sup _{s \in S} w(s) w\left(s^{-1}\right)<\infty .
$$

Now we can formulate the main results of the present note.

Theorem 1.2. Let $\mathcal{G}$ be a locally compact, non-compact group. Suppose that there is a dispersed set $S \subseteq \mathcal{G}$ on which the weight function $w$ is diagonally bounded. Then the following three assertions hold.

(i) $L_{1}(\mathcal{G}, w)$ is strongly Arens irregular in the sense of Dales and Lau (see [1]); i.e., both topological centres of $L_{1}(\mathcal{G}, w)^{* *}$ equal $L_{1}(\mathcal{G}, w)$.

(ii) The topological centre of the algebra $\operatorname{LUC}\left(\mathcal{G}, w^{-1}\right)^{*}$ equals the weighted measure algebra $\mathrm{M}(\mathcal{G}, w)$.

(iii) Every linear (left) $L_{\infty}\left(\mathcal{G}, w^{-1}\right)^{*}$-module map $\Phi \quad: \quad L_{\infty}\left(\mathcal{G}, w^{-1}\right) \rightarrow$ $L_{\infty}\left(\mathcal{G}, w^{-1}\right)$ is automatically bounded, and even $w^{*}-w^{*}$-continuous; hence, $\Phi=\operatorname{conv}(\mu)^{*}$ for some $\mu \in \mathrm{M}(\mathcal{G}, w)$, where $\operatorname{conv}(\mu)(f)=\mu * f(f \in$ $\left.L_{1}(\mathcal{G}, w)\right)$.

Remark 1.3. Let us briefly comment on the above three results.

(1) Assertions (i) and (ii) generalize the main results (Thm. 1 in both cases) of [8] and [7], respectively, from the setting of classical convolution algebras to 
the weighted situation. Although covering a more general case, our proofs are not of higher complexity, if not even simpler, than the ones given in [8] and [7].

We note that (i) and (ii) above have very recently been obtained independently ${ }^{1}$ and via different methods by Dales and Lau in [1] where the present work is mentioned (see [1, Thm. 11.9 and Thm. 12.3] with remark thereafter). To be more precise, (ii) is shown in [1] under the additional assumption that the weight function $w$ satisfies $w \geq 1$, and by an indirect argument, the authors indicate that their proof can be modified to yield (i). Our proofs for both (i) and (ii) are direct and do not assume that $w \geq 1$. We should mention that a detailed proof for (i) is given in [1] under the additional assumption that the compact covering number $\kappa(\mathcal{G})$ is a nonmeasurable cardinal; unfortunately, this proof relies on [1, Lemma 12.1], which is incorrect, as noticed by Zhiguo $\mathrm{Hu}$. This is pointed out in the very recent memoir [2] by Dales, Lau and Strauss.

(2) Part of assertion (iii) shows that the Hofmeier-Wittstock conjecture on automatic boundedness of linear (left) $L_{\infty}(\mathcal{G})^{*}$-module maps on $L_{\infty}(\mathcal{G})$ (cf. [6]) even holds true for Beurling algebras (under a very mild condition on the weight function); it thus establishes a weighted version of the main result in [13], the solution to the original conjecture. Moreover, (iii) generalizes the result, due to Ghahramani-McClure [4], that any bounded linear (left) $L_{\infty}(\mathcal{G})^{*}$-module map on $L_{\infty}(\mathcal{G})$ is automatically $w^{*}$-continuous, to the setting of weighted convolution algebras.

Let us finally mention that our statement (iii) does not appear in [1] - nor does our main tool, the factorization result (Theorem 2.1), which is in fact the key ingredient and unifying element in our approach to all assertions (i), (ii) and (iii).

We wish to emphasize the following important points concerning our approach (cf. also [12]).

- We obtain sharpenings of all statements contained in Theorem 1.2. Indeed, concerning, e.g., (i), we will show that for an element $m \in L_{\infty}\left(\mathcal{G}, w^{-1}\right)^{*}$ to belong to $L_{1}(\mathcal{G}, w)$, it suffices that left multiplication by $m$ be $w^{*}-w^{*}$ continuous on the $w^{*}$-closure of the set of all Hahn-Banach extensions of functionals in $\widetilde{\widetilde{\delta}}_{\mathcal{G}}^{w^{*}} \subseteq \operatorname{Ball}\left(\operatorname{LUC}\left(\mathcal{G}, w^{-1}\right)^{*}\right)$ to $L_{\infty}\left(\mathcal{G}, w^{-1}\right)^{*}$. In contrast, the definition of the topological centre demands $w^{*}$-continuity on all of $L_{\infty}\left(\mathcal{G}, w^{-1}\right)^{*}$. Similar statements hold regarding Theorem 1.2 (ii) and (iii). See Remark 3.8.

- The proofs are direct and follow, once the necessary prerequisites are established (section 2), a general Banach algebraic pattern (section 3).

Finally, we shall demonstrate that our methods not only yield the topological centres of the algebras $L_{1}(\mathcal{G}, w)^{* *}$ and $\operatorname{LUC}\left(\mathcal{G}, w^{-1}\right)^{*}$, but without further effort also the topological centre of the "largest" semigroup compactification of $\mathcal{G}$, i.e., the LUC-compactification $\mathcal{G}^{\mathrm{LUC}}$. Indeed, the simplest case $(w \equiv 1)$ of our factorization result, Theorem 2.1, implies immediately that the topological centre $\Lambda\left(\mathcal{G}^{\mathrm{LUC}} \backslash \mathcal{G}\right)$ of

\footnotetext{
${ }^{1}$ When Dales-Lau and the author found out that we had obtained the same results, we decided to use a common terminology - such as "dispersed set" and "diagonally bounded (weight function)".
} 
the remainder $\mathcal{G}^{\mathrm{LUC}} \backslash \mathcal{G}$ is empty. This is the main result of Protasov-Pym's work [17], which in turn sharpens the main result of Lau-Pym's earlier paper [10], stating that the topological centre of $\mathcal{G}^{\mathrm{LUC}}$ is precisely the group $\mathcal{G}$ (see also [9]). In our approach, the above-mentioned sharpening of statement (ii) in our Theorem 1.2 is crucial: indeed, it allows us to arrange the proof of the fact that $\Lambda\left(\mathcal{G}^{\mathrm{LUC}} \backslash \mathcal{G}\right)=\varnothing$ in a way that partially answers a problem raised in [10]; see Remark 3.10 below. Let us recall here that the compact semitopological semigroup $\mathcal{G}^{\mathrm{LUC}}$ may be identified with the spectrum of the commutative $C^{*}$-algebra $\operatorname{LUC}(\mathcal{G})$. Note that in the case where $\mathcal{G}$ is discrete, $\mathcal{G}^{\mathrm{LUC}}$ is nothing but the Stone-Čech compactification $\beta \mathcal{G}$ (equipped with the appropriate multiplication). The topological centre of $\mathcal{G}^{\mathrm{LUC}}$ is defined as

$$
\Lambda\left(\mathcal{G}^{\mathrm{LUC}}\right):=\left\{m \in \mathcal{G}^{\mathrm{LUC}} \mid n \mapsto m n \text { is } w^{*} \text {-w*-continuous on } \mathcal{G}^{\mathrm{LUC}}\right\} .
$$

The interesting part of $\mathcal{G}^{\mathrm{LUC}}$, the so-called remainder $\mathcal{G}^{\mathrm{LUC}} \backslash \mathcal{G}$, is itself a compact semitopological semigroup, and its topological centre is defined analogously to the above.

\section{A faCtorization theOREM For families of FUnCtions in $L_{\infty}\left(\mathcal{G}, w^{-1}\right)$}

We shall now present our principal tool, which is a general factorization theorem for bounded families in $L_{\infty}\left(\mathcal{G}, w^{-1}\right)$. It provides a generalization of Thm. 2.1 in [13] to the weighted setting and is of interest in its own right.

Theorem 2.1. Let $\mathcal{G}$ be a locally compact, non-compact group. Suppose that there is a dispersed set $S \subseteq \mathcal{G}$ on which the weight function $w$ is diagonally bounded. Then there exists a family $\left(\psi_{\alpha}\right)_{\alpha \in I},|I|=\kappa(\mathcal{G})$, of functionals in $\overline{\widetilde{\delta}}_{\mathcal{G}} w^{*} \subseteq$ $\operatorname{Ball}\left(\operatorname{LUC}\left(\mathcal{G}, w^{-1}\right)^{*}\right)$ such that whenever $\left(h_{\alpha}\right)_{\alpha \in I} \subseteq L_{\infty}\left(\mathcal{G}, w^{-1}\right)$ is a bounded family of functions, there exists a function $h \in L_{\infty}\left(\mathcal{G}, w^{-1}\right)$ such that the factorization formula

holds for all $\alpha \in I$.

$$
h_{\alpha}=\psi_{\alpha} \diamond h
$$

Proof. For $y \in \mathcal{G}$, we denote by $r_{y}$ the operator of right translation; i.e., $\left(r_{y} f\right)(x)=$ $f(x y)$ whenever $f$ is a function on $\mathcal{G}$ and $x \in \mathcal{G}$.

There is a covering of $\mathcal{G}$ by open sets whose closure is compact, of minimal cardinality, i.e., of cardinality $\kappa(\mathcal{G})$, and closed under finite unions; we denote the corresponding family of compacta by $\left(K_{\alpha}\right)_{\alpha \in I}$. Set $\widetilde{I}:=I \times I$. For $\widetilde{\alpha}=(\alpha, i) \in$ $\widetilde{I}$, put $K_{\widetilde{\alpha}}=K_{(\alpha, i)}:=K_{\alpha}$. Then $\left(K_{\widetilde{\alpha}}\right)_{\widetilde{\alpha} \in \widetilde{I}}$ is a covering of $\mathcal{G}$ having the same properties as the original one. Since the set $S$ is dispersed, by the same reasoning as in Lemma 3 of [8], we see that there exists a family $\left(y_{\widetilde{\alpha}}\right)_{\widetilde{\alpha} \in \widetilde{I}} \subseteq S$ such that

$$
K_{\widetilde{\alpha}} y_{\widetilde{\alpha}}^{-1} \cap K_{\widetilde{\beta}} y_{\widetilde{\beta}}^{-1}=\varnothing \quad \forall \widetilde{\alpha}, \widetilde{\beta} \in \widetilde{I}, \widetilde{\alpha} \neq \widetilde{\beta} .
$$

Set $S^{\prime}:=\left\{y_{\widetilde{\alpha}} \mid \widetilde{\alpha} \in \widetilde{I}\right\}$. We define, for $(\alpha, i),(\beta, j) \in \widetilde{I}$ :

$$
(\alpha, i) \preceq(\beta, j): \Longleftrightarrow K_{(\alpha, i)} \subseteq K_{(\beta, j)} \Longleftrightarrow K_{\alpha} \subseteq K_{\beta} \Longleftrightarrow: \alpha \preceq^{\prime} \beta .
$$

Let $\mathfrak{F}$ be an ultrafilter on $I$ which dominates the order filter. Define, for $j \in I$,

$$
\psi_{j}{ }^{\prime}:=w^{*}-\lim _{\beta \rightarrow \mathfrak{F}} \widetilde{\delta}_{y_{(\beta, j)}^{-1}} \in \widetilde{\widetilde{\delta}}_{\mathcal{G}}^{w^{*}} \subseteq \operatorname{Ball}\left(\operatorname{LUC}\left(\mathcal{G}, w^{-1}\right)^{*}\right)
$$

and let $\psi_{j}$ be arbitrary Hahn-Banach extensions of $\psi_{j}{ }^{\prime}$ to $L_{\infty}\left(\mathcal{G}, w^{-1}\right)^{*}$. 
Since $w$ is diagonally bounded on $S^{\prime}$, we have:

$$
\sup _{s \in S^{\prime}}\left\|w\left(s^{-1}\right) \delta_{s}\right\|_{w}=\sup _{s \in S^{\prime}} w(s) w\left(s^{-1}\right)<\infty .
$$

Thus, the family of functions

$$
H_{(\alpha, i)}:=\left(w\left(y_{(\alpha, i)}^{-1}\right) \delta_{y_{(\alpha, i)}}\right) \diamond\left(\chi_{K_{(\alpha, i)}} h_{i}\right)=w\left(y_{(\alpha, i)}^{-1}\right) r_{y_{(\alpha, i)}}\left(\chi_{K_{(\alpha, i)}} h_{i}\right)
$$

is bounded in $L_{\infty}\left(\mathcal{G}, w^{-1}\right)$, whence $\left(w^{-1} H_{(\alpha, i)}\right)$ is a bounded family in $L_{\infty}(\mathcal{G})$. By (1), the projections $r_{y_{(\alpha, i)}} \chi_{K_{(\alpha, i)}}=\chi_{K_{(\alpha, i)} y_{(\alpha, i)}^{-1}}$ are pairwise orthogonal, so that

$$
H:=\sum_{\alpha \in I} \sum_{i \in I} w^{-1} H_{(\alpha, i)} \quad\left(w^{*} \text {-limits }\right)
$$

defines a function in $L_{\infty}(\mathcal{G})$. Hence, we have

$$
h:=\sum_{\alpha \in I} \sum_{i \in I} H_{(\alpha, i)} \in L_{\infty}\left(\mathcal{G}, w^{-1}\right) .
$$

Using (1), we obtain for all $(\alpha, i),(\beta, j),(\gamma, k) \in \widetilde{I}$ with $(\gamma, k) \preceq(\beta, j)$ :

$$
\begin{aligned}
\chi_{K_{(\gamma, k)}} & r_{y_{(\beta, j)}^{-1}} r_{y_{(\alpha, i)}}\left(\chi_{K_{(\alpha, i)}} h_{i}\right) \\
= & \chi_{K_{(\gamma, k)}} \chi_{K_{(\beta, j)}} r_{y_{(\beta, j)}^{-1}} r_{y_{(\alpha, i)}}\left(\chi_{K_{(\alpha, i)}} h_{i}\right) \\
= & \chi_{K_{(\gamma, k)}}\left[r_{y_{(\beta, j)}^{-1}}\left(\left(r_{y_{(\beta, j)}} \chi_{K_{(\beta, j)}}\right) r_{y_{(\alpha, i)}}\left(\chi_{K_{(\alpha, i)}} h_{i}\right)\right)\right] \\
= & \delta_{(\alpha, i),(\beta, j)} \chi_{K_{(\gamma, k)}} h_{j} .
\end{aligned}
$$

Taking into account (2), we deduce that for all $j \in I$ and $(\gamma, k) \in \widetilde{I}$ :

$$
\begin{aligned}
\chi_{K_{(\gamma, k)}}\left(\psi_{j} \diamond h\right) \\
=w^{*}-\lim _{\beta \rightarrow \mathfrak{F}} \sum_{\alpha \in I} \sum_{i \in I} w\left(y_{(\alpha, i)}^{-1}\right) w\left(y_{(\beta, j)}^{-1}\right)^{-1} \underbrace{\chi_{K_{(\gamma, k)}} r_{y_{(\beta, j)}^{-1}} r_{y_{(\alpha, i)}}\left(\chi_{K_{(\alpha, i)}} h_{i}\right)}_{=\delta_{(\alpha, i),(\beta, j)} \chi_{K_{(\gamma, k)}} h_{j}} \\
=\chi_{K_{(\gamma, k)} h_{j},}
\end{aligned}
$$

whence the desired factorization formula follows.

\section{The topological Centres of $L_{1}(\mathcal{G}, w)^{* *}$ And $\operatorname{LUC}\left(\mathcal{G}, w^{-1}\right)^{*}$}

We shall first briefly recall the following definitions from [15] and [14], respectively.

Definition 3.1. Let $X$ be a Banach space and $\kappa \geq \aleph_{0}$ a cardinal number. We say that $X$ has the Mazur property of level $\kappa$ [property $\left(M_{\kappa}\right)$, for short] if every $w^{*}$ - $\kappa$-continuous functional $f \in X^{* *}$ belongs to $X$.

Here, $f \in X^{* *}$ is called $w^{*}-\kappa$-continuous if for all nets $\left(x_{\alpha}\right)_{\alpha \in I} \subseteq \operatorname{Ball}\left(X^{*}\right)$ of cardinality $\aleph_{0} \leq|I| \leq \kappa$ which converge $w^{*}$ to 0 , we have that $\left\langle f, x_{\alpha}\right\rangle \rightarrow 0$.

Definition 3.2. Let $\mathcal{A}$ be a Banach algebra and $\kappa$ a cardinal number. Then $\mathcal{A}^{*}$ is said to have

(i) the left $\mathcal{A}^{* *}$ factorization property of level $\kappa$ [property $\left(F_{\kappa}^{l}\right)$, for short] if for any family of functionals $\left(h_{\alpha}\right)_{\alpha \in I} \subseteq \operatorname{Ball}\left(\mathcal{A}^{*}\right)$ with $|I|=\kappa$, there exist 
a family $\left(\psi_{\alpha}\right)_{\alpha \in I} \subseteq \operatorname{Ball}\left(\mathcal{A}^{* *}\right)$ and one single functional $h \in \mathcal{A}^{*}$ such that the factorization

holds for all $\alpha \in I$;

$$
h_{\alpha}=\psi_{\alpha} \odot h
$$

(ii) the left uniform $\mathcal{A}^{* *}$ factorization property of level $\kappa$ [property $\left(U F_{\kappa}^{l}\right)$, for short] if there is a family $\left(\psi_{\alpha}\right)_{\alpha \in I} \subseteq \operatorname{Ball}\left(\mathcal{A}^{* *}\right)$ with $|I|=\kappa$, such that for any family of functionals $\left(h_{\alpha}\right)_{\alpha \in I} \subseteq \operatorname{Ball}\left(\mathcal{A}^{*}\right)$, there is one single functional $h \in \mathcal{A}^{*}$ such that the factorization

$$
h_{\alpha}=\psi_{\alpha} \odot h
$$

holds for all $\alpha \in I$.

Remark 3.3. Obviously, we also have "right" versions $\left(F_{\kappa}^{r}\right)$ and $\left(U F_{\kappa}^{r}\right)$ of the above factorization properties. If $\mathcal{A}^{*}$ enjoys both the left and right factorization properties, we say that it has property $\left(F_{\kappa}\right)$, respectively, $\left(U F_{\kappa}\right)$.

Let us now consider these properties in our situation of Beurling algebras.

Proposition 3.4. For an arbitrary locally compact group $\mathcal{G}$, the space $L_{1}(\mathcal{G}, w)$ enjoys property $M_{\kappa(\mathcal{G}) \cdot \aleph_{0}}$.

Proof. This follows from Thm. 3.4 in [15], which states the above property for the space $L_{1}(\mathcal{G})$, and the fact that the property is stable under isomorphisms (cf. [15, Remark 3.3]).

The following is an immediate consequence of our Theorem 2.1 above (by simply taking Hahn-Banach extensions to $L_{\infty}\left(\mathcal{G}, w^{-1}\right)^{*}$ of the functionals $\psi_{\alpha}$ in $\overline{\widetilde{\delta}}_{\mathcal{G}}^{w^{*}} \subseteq$ Ball $\left(\operatorname{LUC}\left(\mathcal{G}, w^{-1}\right)^{*}\right)$ obtained there).

Corollary 3.5. Let $\mathcal{G}$ be a locally compact, non-compact group. Suppose that there is a dispersed set $S \subseteq \mathcal{G}$ on which the weight function $w$ is diagonally bounded. Then $L_{\infty}\left(\mathcal{G}, w^{-1}\right)$ has property $U F_{\kappa(\mathcal{G})}$.

Remark 3.6. A straightforward modification of the proof of our Theorem 2.1 above yields a corresponding right factorization result for $\operatorname{RUC}\left(\mathcal{G}, w^{-1}\right)^{*}$ from which one readily deduces that $L_{\infty}\left(\mathcal{G}, w^{-1}\right)$ has property $\left(U F_{\kappa(\mathcal{G})}^{r}\right)$; hence $L_{\infty}\left(\mathcal{G}, w^{-1}\right)$ has property $\left(U F_{\kappa(\mathcal{G})}\right)$.

With the above preparations in place, our Theorem 1.2 will be an immediate consequence of the following general Banach algebraic result.

Theorem 3.7. Let $\mathcal{A}$ be a Banach algebra satisfying $\left(M_{\kappa}\right)$ and whose dual $\mathcal{A}^{*}$ has the property $\left(F_{\kappa}\right)$, for some $\kappa \geq \aleph_{0}$. Then the following three statements hold. ${ }^{2}$

(i) The Banach algebra $\mathcal{A}$ is strongly Arens irregular.

(ii) If $\mathcal{A}$ has a right approximate identity bounded by 1 , then the topological centre of $\left(\mathcal{A}^{*} \odot \mathcal{A}\right)^{*}$ can be identified (up to isometric algebra isomorphism) with the algebra $\operatorname{RM}(\mathcal{A})$ of right multipliers of $\mathcal{A}$; i.e., $\mathfrak{Z}_{t}\left(\left(\mathcal{A}^{*} \odot \mathcal{A}\right)^{*}\right) \cong$ $\operatorname{RM}(\mathcal{A})$

(iii) Every linear left $\mathcal{A}^{* *}$-module homomorphism on $\mathcal{A}^{*}$ is automatically bounded and even $w^{*}$-w $w^{*}$-continuous; i.e., $\mathcal{L}_{\mathcal{A}^{* *}}\left(\mathcal{A}^{*}\right)=\mathcal{B}_{\mathcal{A}^{* *}}^{\sigma}\left(\mathcal{A}^{*}\right)$.

\footnotetext{
${ }^{2}$ In (ii) and (iii), it is enough to assume $\left(F_{\kappa}^{l}\right)$ instead of $\left(F_{\kappa}\right)$.
} 
Proof. This follows from Theorems 2.3 and 4.2 in [14].

Let us now deduce Theorem 1.2.

Proof. In view of Theorem 2.1, Proposition 3.4 and Corollary 3.5 (together with Remark 3.6), we can apply Theorem 3.7 to $\mathcal{A}=L_{1}(\mathcal{G}, w)$. For (ii), note that, as is easily seen, $L_{1}(\mathcal{G}, w)$ always has a bounded approximate identity of norm 1 . Also, by a weighted version of Wendel's classical result, as given in [3, Lemma 2.3] with an elegant proof, we have $R M\left(L_{1}(\mathcal{G}, w)\right)=M(\mathcal{G}, w)$. Finally, the last assertion in Theorem 1.2 (iii), i.e., the fact that any linear $w^{*}$-w*-continuous (left) $L_{\infty}\left(\mathcal{G}, w^{-1}\right)^{*}$-module map $\Phi: L_{\infty}\left(\mathcal{G}, w^{-1}\right) \rightarrow L_{\infty}\left(\mathcal{G}, w^{-1}\right)$ is of the form $\Phi=$ $\operatorname{conv}(\mu)^{*}$ for some $\mu \in \mathrm{M}(\mathcal{G}, w)$, is easily seen as follows: one first checks that $\Phi_{*}$ is a left multiplier (i.e., a right $L_{1}(\mathcal{G}, w)$-module map) on $L_{1}(\mathcal{G}, w)$ (cf. the proof of [13, Corollary 3.8]); then [3, Lemma 2.3] implies that $\Phi_{*}=\operatorname{conv}(\mu)$ for some $\mu \in \mathrm{M}(\mathcal{G}, w)$.

Remark 3.8. Inspection of the proofs of Theorems 2.3 and 4.2 in [14] shows that to ensure the assertions of Theorem 3.7, it is enough to assume the topological centre condition (in (i), (ii)) and the module property (in (iii)), respectively, only with respect to elements from the set ${\overline{\left\{\psi_{\alpha} \mid \alpha \in I\right\}}}^{w^{*}} \subseteq \mathcal{A}^{* *}$ and the set ${\overline{\left\{\psi_{\alpha}\left|\mathcal{A}^{*} \odot \mathcal{A}\right| \alpha \in I\right\}}}^{w^{*}} \subseteq\left(\mathcal{A}^{*} \odot \mathcal{A}\right)^{*}$, respectively; here, $\left(\psi_{\alpha}\right)_{\alpha \in I}$ is the family of "factoring" functionals pertaining to property $\left(F_{\kappa}\right)$.

We now show how the easiest case, namely $w \equiv 1$, in our Theorem 2.1 may be used to derive the result, due to Protasov and Pym [17], on the extreme discontinuity of multiplication in the remainder $\mathcal{G}^{\mathrm{LUC}} \backslash \mathcal{G}$.

Theorem 3.9. Let $\mathcal{G}$ be a locally compact group. Then $\Lambda\left(\mathcal{G}^{\mathrm{LUC}} \backslash \mathcal{G}\right)=\varnothing$.

Proof. To avoid trivialities, we suppose that $\mathcal{G}$ is non-compact. Assume towards a contradiction that there is $m \in \Lambda\left(\mathcal{G}^{\mathrm{LUC}} \backslash \mathcal{G}\right)$. Since $\mathcal{G}=\mathrm{M}(\mathcal{G}) \cap \mathcal{G}^{\mathrm{LUC}}$, we only need to show that $m \in \mathrm{M}(\mathcal{G})$. As noted in Remark 3.8 with regard to Theorem 3.7 (ii) (applied to $\mathcal{A}=L_{1}(\mathcal{G})$ ), if for $m \in \operatorname{LUC}(\mathcal{G})^{*}$ the map $n \mapsto m n$ is $w^{*}$-w*-continuous at all points $n \in \mathcal{N}:=\overline{\left\{\left.\psi_{\alpha}\right|_{\mathrm{LUC}(\mathcal{G})} \mid \alpha \in I\right\}} w^{*} \subseteq \mathrm{LUC}(\mathcal{G})^{*}$, then $m \in \mathrm{M}(\mathcal{G})$; cf. also the first remark on page 166 of [12]. Obviously, in our situation, $\mathcal{N} \subseteq \mathcal{G}^{\text {LUC }} \backslash \mathcal{G}$. But then, since $m \in \Lambda\left(\mathcal{G}^{\mathrm{LUC}} \backslash \mathcal{G}\right)$, we have $m \in \mathrm{M}(\mathcal{G})$, as desired.

Remark 3.10. In [10], the authors note that, since $\mathfrak{Z}_{t}\left(\mathrm{LUC}(\mathcal{G})^{*}\right)=\mathrm{M}(\mathcal{G})$, it is "tempting to suppose that, as a consequence," $\Lambda\left(\mathcal{G}^{\mathrm{LUC}}\right)=\mathrm{M}(\mathcal{G}) \cap \mathcal{G}^{\mathrm{LUC}}=\mathcal{G}$, but that they "have been unable to find a way of making this deduction". As the above proof shows, our sharpening of the result $\mathfrak{Z}_{t}\left(\mathrm{LUC}(\mathcal{G})^{*}\right)=\mathrm{M}(\mathcal{G})$ indeed immediately yields the second one. Hence, we suggest that both statements be seen as consequences of the one result that for any element $m \in \operatorname{LUC}(\mathcal{G})^{*}$, the $w^{*}-w^{*}$ continuity of left multiplication by $m$ on $\mathcal{G}^{\mathrm{LUC}}$ is enough to ensure that $m \in \mathrm{M}(\mathcal{G})$, which, in the case where $m$ happens to belong to $\mathcal{G}^{\mathrm{LUC}}$, of course means that $m$ is a point mass. 


\section{REFERENCES}

[1] Dales, H. G.; LAU, A. T.-M., The second duals of Beurling algebras, Mem. Amer. Math. Soc. 177 (2005), no. 836. MR2155972 (2006k:43002)

[2] Dales, H. G.; Lau, A. T.-M.; Strauss, D., Banach algebras on semigroups and their compactifications, preprint, submitted to the Memoirs of the American Mathematical Society.

[3] Ghahramani, F., Weighted group algebra as an ideal in its second dual space, Proc. Amer. Math. Soc. 90 (1984), no. 1, 71-76. MR722417 (85i:43007)

[4] Ghahramani, F.; McClure, J.P., Module homomorphisms of the dual modules of convolution Banach algebras, Canad. Math. Bull. 35 (1992), no. 2, 180-185. MR1165166 (93f:43004)

[5] Grønbeк, N., Amenability of weighted convolution algebras on locally compact groups, Trans. Amer. Math. Soc. 319 (1990), no. 2, 765-775. MR962282 (90j:43003)

[6] Hofmeier, H.; Wittstock, G., A bicommutant theorem for completely bounded module homomorphisms, Math. Ann. 308 (1997), no. 1, 141-154. MR1446204 (98h:46065)

[7] LAU, A. T.-M., Continuity of Arens multiplication on the dual space of bounded uniformly continuous functions on locally compact groups and topological semigroups, Math. Proc. Camb. Phil. Soc. 99 (1986), 273-283. MR817669 (87i:43001)

[8] LaU, A. T.-M.; Losert, V., On the second conjugate algebra of $L_{1}(\mathcal{G})$ of a locally compact group, J. London Math. Soc. (2) 37 (1988), no. 3, 464-470. MR939122 (89e:43007)

[9] Lau, A. T.-M.; Milnes, P.; PyM, J. S., Locally compact groups, invariant means and the centres of compactifications, J. London Math. Soc. (2) 56 (1997), no. 1, 77-90. MR1462827 (98k:22021)

[10] LaU, A. T.-M.; PYM, J., The topological centre of a compactification of a locally compact group, Math. Z. 219 (1995), no. 4, 567-579. MR1343662 (96e:22010)

[11] LAU, A. T.-M.; Ülger, A., Topological centers of certain dual algebras, Trans. Amer. Math. Soc. 348 (1996), no. 3, 1191-1212. MR1322952 (96h:43003)

[12] Neufang, M., A unified approach to the topological centre problem for certain Banach algebras arising in abstract harmonic analysis, Arch. Math. 82 (2004), no. 2, 164-171. MR2047670 (2005g:22004)

[13] Neufang, M., Solution to a conjecture by Hofmeier-Wittstock, J. Funct. Anal. 217 (2004), no. 1, 171-180. MR2097611 (2005i:43003)

[14] Neufang, M., On a conjecture by Ghahramani-Lau and related problems concerning topological centres, J. Funct. Anal. 224 (2005), no. 1, 217-229. MR2139110 (2006b:46063)

[15] Neufang, M., On the Mazur property and property $(X)$, to appear in: Journal of Operator Theory.

[16] Palmer, T. W., Banach algebras and the general theory of ${ }^{*}$-algebras. Vol. I. Algebras and Banach algebras, Encyclopedia of Mathematics and its Applications, 49. Cambridge University Press, Cambridge, 1994. MR1270014 (95c:46002)

[17] Protasov, I. V.; Pym, J. S., Continuity of multiplication in the largest compactification of a locally compact group, Bull. London Math. Soc. 33 (2001), no. 3, 279-282. MR1817766 (2002h:22006)

School of Mathematics and Statistics, Carleton University, Ottawa, Ontario, K1S 5B6 CANADA

E-mail address: mneufang@math.carleton.ca 